\title{
Animated Faces for Robotic Heads: Gaze and Beyond
}

\author{
Samer Al Moubayed, Jonas Beskow, Jens Edlund, Björn Granström, and David \\ House \\ Department of Speech, Music and Hearing. KTH Royal Institute of Technology, \\ Stockholm, Sweden \\ sameram@kth.se, beskow@kth.se, edlund@speech.kth.se, bjorn@speech.kth.se, \\ davidh@kth.se \\ http://www.speech.kth.se
}

\begin{abstract}
We introduce an approach to using animated faces for robotics where a static physical object is used as a projection surface for an animation. The talking head is projected onto a $3 \mathrm{D}$ physical head model. In this chapter we discuss the different benefits this approach adds over mechanical heads. After that, we investigate a phenomenon commonly referred to as the Mona Lisa gaze effect. This effect results from the use of $2 \mathrm{D}$ surfaces to display 3D images and causes the gaze of a portrait to seemingly follow the observer no matter where it is viewed from. The experiment investigates the perception of gaze direction by observers. The analysis shows that the 3D model eliminates the effect, and provides an accurate perception of gaze direction. We discuss at the end the different requirements of gaze in interactive systems, and explore the different settings these findings give access to.
\end{abstract}

Keywords: Facial Animation, Talking Heads, Shader Lamps, Robotic Heads, Gaze, Mona Lisa Effect, Avatar, Dialogue System, Situated Interaction, 3D Projection, Gaze Perception.

\section{Introduction}

During the last two decades, there has been ongoing research and impressive enhancement in facial animation. Researchers have been developing human-like talking heads that can have human-like interaction with humans [1], realize realistic facial expressions [2], and express emotions [3] and communicate behaviors [4].

Several talking heads are made to represent personas embodied in 3D facial designs (referred to as ECAs, Embodied Conversational Agents) simulating human behavior and establishing interaction and conversation with a human interlocutor. Although these characters have been embodied in human-like 3D animated models, this embodiment has always been limited by how these characters are displayed in our environment. Traditionally, talking heads have been displayed using two dimensional display (e.g. flat screens, wall projections, etc) 
having no shared access to the three dimensional environment where the interaction is taking place. Surprisingly, there is little research on the effects of displaying 3D ECAs on 2D surfaces on the perception of the agent embodiment and its natural interaction effects [5]. Moreover, 2D displays come with several usually undesirable illusions and effects, such as the Mona Lisa gaze effect. For a review on these effects, refer to [6].

In robotics on the other hand, the complexity, robustness and high resolution of facial animation, which is done using computer graphics, is not employed. This is due to the fact that the accurate and highly subtle and complicated control of computer models (such as eyes, eye-lids, wrinkles, lips, etc.) do not map onto mechanically controlled heads. Such computer models require very delicate, smooth, and fast control of the motors, appearance and texture of a mechanical head. This fact has a large implication on the development of robotic heads. Moreover, in a physical mechanical robot head, the design and implementation of anthropomorphic properties can be limited, highly expensive, time consuming and difficult to test until the final head is finished. In talking heads on the other hand, changes in color, design, features, and even control of the face can be very easy and time efficient compared to mechanically controlled heads.

There are few studies attempting to take advantage of the appearance and behavior of talking heads in the use of robotic heads. In [7], a flat screen is used as the head of the robot, displaying an animated agent. In [8], the movements of the motors of a mechanical head are driven by the control parameters of animated agents, in a trial to generate facial trajectories that are similar to those of a 3D animated face. These studies, although showing the interest and need to use the characteristics of animated talking agents in robot heads, are still limited by how this agent is represented, in the first case by a $2 \mathrm{D}$ screen that comes with detrimental effects and illusions, but profits from the appearance of the animated face, and in the second case by a mechanical head that tries to benefit from the behavior but misses on appearance.

In this chapter we will present a new approach for using animated faces for robotic heads, where we attempt to guarantee the physical dimensionality and embodiment of the robotic head, and the appearance and behavior of the animated agents. After representing our approach and discussing its benefits, we investigate and evaluate this approach by studying its accuracy in delivering gaze direction in comparison to two dimensional display surfaces.

Perhaps one of the most important effects of displaying three-dimensional scenes on two-dimensional surfaces is the Mona Lisa gaze effect. The Mona Lisa gaze effect is commonly described as an effect that makes it appear as if the Mona Lisas gaze rests steadily on the viewer as the viewer moves through the room. This effect has important implications in situational and spatial interaction, since gaze direction of a face displayed over a two-dimensional display does not point to an absolute location in the environment of the observer.

In Section 2 we describe our proposal of using a 3D model of a human head as a projection surface for an animated talking head. In Section 3 we discuss 

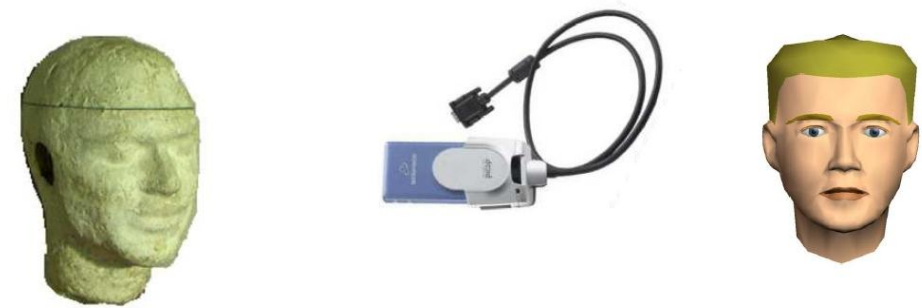

Fig. 1. The technical setup: the physical model of a human head used as a $3 D$ projection surface, to the left; the laser projector in the middle; and a snapshot of the $3 D$ talking head to the right.

the benefits of using our approach in comparison to a traditional mechanical robotic head. In Section 4 we describe an experimental setup and a user study on the perception of gaze targets using a traditional 2D display and the novel $3 \mathrm{D}$ projection surface. In Section 5 we discuss the properties of gaze in terms of faithfulness for different communication requirements and configurations. We discuss different applications that can capitalize on our approach as well as research and experimentation made possible by it in Section 6 and present final conclusions in Section 7.

\section{Projected Animated Faces on 3D Head Models}

Our approach is based on the idea of projecting an animated face on a 3D surface a static, physical model of a human head. The technique of manipulating static objects with light is commonly referred to as the Shader Lamps technique [9] [10]. This technique is used to change the physical appearance of still objects, by illuminating them, using projections of static or animated textures, or video streams. We implement this technique by projecting an animated talking head (seen to the right in figure 1) on an arbitrary physical model of a human head (seen to the left in figure 1) using a laser micro projector (SHOWWX Pico Projector, seen in the center of figure 1). The main advantage of using a laser projector is that the image is always in focus, even on curved surfaces.

The talking head used in the studies is detailed in [11] and includes a face, eyes, tongue, and teeth, based on static 3D-wireframe meshes that are deformed using direct parameterizations by applying weighted transformations to their vertices according to principles first introduced by [12]. Figure 2 shows the 3D projection surface with and without a projection of the talking head.

\section{Robotic Heads with Animated Faces}

the capacity for adequate interaction is a key concern. Since a great proportion of human interaction is managed non-verbally through gestures, facial expressions 

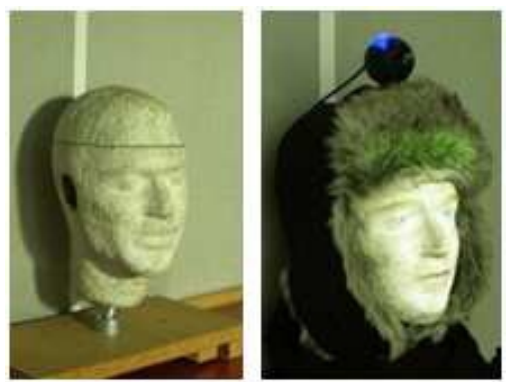

Fig. 2. A physical model of a human head, without projection (left) and complete with a projection of the talking head, a furry hat, and a camera (right).

and gaze, an important current research trend in robotics deals with the design of social robots. But what mechanical and behavioral compromises should be considered in order to achieve satisfying interaction with human interlocutors? In the following, we present an overview of the practical benefits of using an animated talking head projected on a 3D surface as a robotic head.

1 Optically based. Since the approach utilizes a static 3D projection surface, the actual animation is done completely using computer graphics projected on the surface. This provides an alternative to mechanically controlled faces, saving electrical consumption and avoiding complex mechanical designs and motor control. Computer graphics also offers many advantages over motor based animation of robotic heads in speed, animation accuracy, resolution and flexibility.

2 Animation using computer graphics. Facial animation technology has shown tremendous progress over the last decade, and currently offers realistic, efficient, and reliable renditions. It is currently able to establish facial designs that are very human-like in appearance and behavior compared to the physical designs of mechanical robotic heads.

3 Facial design. The face design is done through software, which potentially provides the flexibility of having an unlimited range of facial designs for the same head. Even if the static projection surface needs to be re-customized to match a particularly unusual design, this is considerably simpler, faster, and cheaper than redesigning a whole mechanical head. In addition, the easily interchangeable face design offers the possibility to efficiently experiment with the different aspects of facial designs and characteristics in robotics heads, for example to examine the anthropomorphic spectrum.

4 Light weight. The optical design of the face leads to a considerably more lightweight head, depending only on the design of the projection surface. This makes the design of the neck much simpler and a more light-weight neck can be used, as it has to carry and move less weight. Ultimately, a lighter mobile robot is safer and saves energy.

5 Low noise level. The alternative of using light projection over a motorcontrolled face avoids all motor noises generated by moving the face. This is 
crucial for a robot interacting verbally with humans, and in any situation where noise generation is a problem.

6 Low maintenance. Maintenance is reduced to software maintenance and maintenance of the micro laser projector, which is very easily replaceable. In contrast, mechanical faces are complicated, both electronically and mechanically, and an error in the system can be difficult and time consuming to troubleshoot.

Naturally, there are drawbacks as well. Some robotic face designs cannot be achieved in full using light-projected animation alone, for example those requiring very large jaw openings which cannot be easily and realistically delivered without mechanically changing the physical projection surface. For such requirements, a hybrid approach can be implemented which combines a motor based physical animation of the head for the larger facial movements, with an optically projected animation for the more subtle movements, for example changes in eyes, wrinkles and eyebrows.

In addition, the animations are delivered using light, so the projector must be able to outshine the ambient light, which becomes an issue if the robot is designed to be used in very bright light, such as full daylight. The problem can be remedied by employing the evermore powerful laser projectors that are being brought to the market.

\section{Gaze perception and the Mona Lisa gaze effect}

The importance of gaze in social interaction is well-established. From a human communication perspective, Kendons work in [13] on gaze direction in conversation is particularly important in inspiring a wealth of studies that singled out gaze as one of the strongest non-vocal cues in human face-to-face interaction (see e.g. [14]). Gaze has been associated with a variety of functions within social interaction Kleinkes review article from 1986, for example, contains the following list: (a) provide information, (b) regulate interaction, (c) express intimacy, (d) exercise social control, and (e) facilitate service and task goals ([15]). These efforts, in turn, were shadowed by a surge of activity in the human-computer interaction community, which recognized the importance of modeling gaze in artificial personas such as embodied conversational agents (ECAs) (e.g. [16]; [17]).

To date, these efforts have been somewhat biased towards the production of gaze behavior, whereas less effort has been expended on the perception of gaze. In light of the fact that an overwhelming majority of ECAs are either $2 \mathrm{D}$ or 3D models, rendered on 2D displays, this is somewhat surprising: the perception of $2 \mathrm{D}$ renditions of $3 \mathrm{D}$ scenes is notoriously riddled with artefact and illusions of many sorts for an overview, see [18]. Perhaps the most important of these for using gaze behaviors in ECAs for communicative purposes is the Mona Lisa gaze effect or the Mona Lisa stare, commonly described as an effect that makes it appear as if the Mona Lisas gaze rests steadily on the viewer as the viewer moves through the room (figure 3).

The fact that the Mona Lisa gaze effect occurs when a face is presented on a $2 \mathrm{D}$ display has significant consequences for the use and control of gaze in 


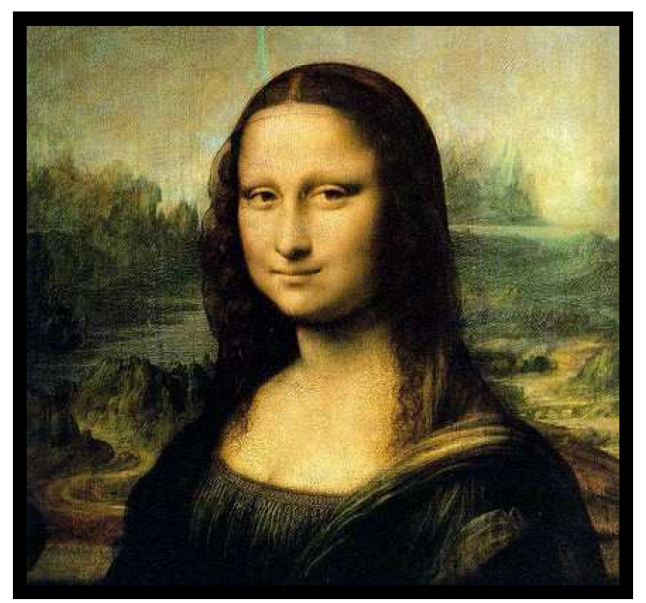

Fig. 3. Leonardo da Vinci's Mona Lisa. Mona Lisa appears to be looking straight at the viewer, regardless of viewing angle. The painting is in the public domain.

communication. To the extent that gaze in a $2 \mathrm{D}$ face follows the observer, gaze does not point unambiguously at a point in $3 \mathrm{D}$ space. In the case of multiple observers, they all have the same perception of the image, no matter where they stand in relation to e.g. the painting or screen. This causes an inability to establish a situated eye contact with one particular observer, without simultaneously establishing it with all others, which leads to miscommunication if gaze is employed to support a smooth flowing interaction with several human subjects: all human subjects will perceive the same gaze pattern.

In the following experiment, we investigate the accuracy of perceived gaze direction in our 3D head model, discuss the different applications it can be used for, and contrast it with a traditional $2 \mathrm{D}$ display.

The experiment detailed here was designed and conducted to confirm the hypothesis that a talking head projected on a $2 \mathrm{D}$ display is subject to the Mona Lisa gaze effect, while projecting it on a $3 \mathrm{D}$ surface inhibits the effect and enforces an eye-gaze direction that is independent of the subjects angle of view. Accordingly, the experiment measures perception accuracy of gaze in these two configurations.

\subsection{Setup}

The experiment setup employs a set of subjects simultaneously seated on a circle segment centrad at the stimulus point a $2 \mathrm{D}$ or $3 \mathrm{D}$ projection surface facing the stimuli point. Adjacent subjects are equidistant from each other and all subjects are equidistant to the projection surface so that the angle between two adjacent subjects and the projection surface was always about 26.5 degrees. The positions are annotated as $-53,-26.5,0,26.5,53$, where 0 is the seat directly in front of 


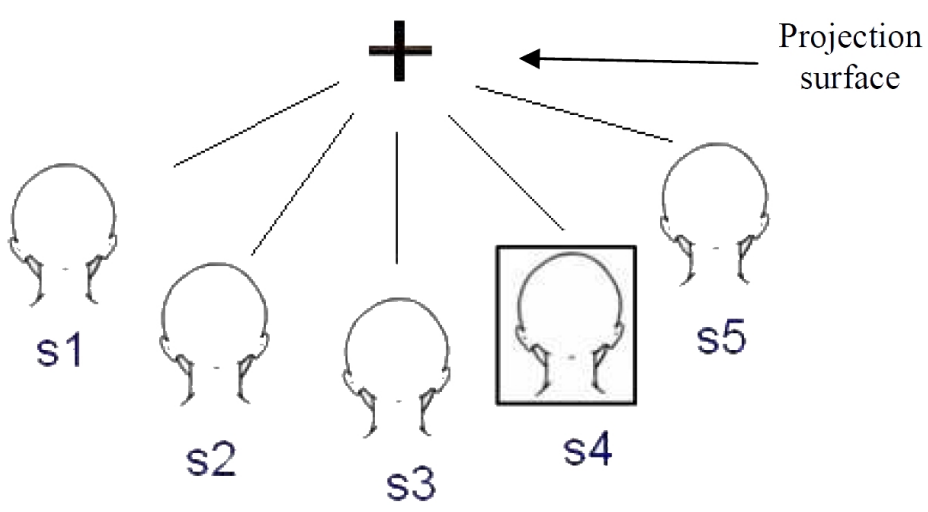

Fig. 4. Schematic of the experiment setup: five simultaneous subjects are placed at equal distances along the perimeter of a circle centred on the projection surface

the projection surface. The distance from subjects to the projection surface was 1.80 meters (figure 4).

Two identical sets of stimuli are projected on a $2 \mathrm{D}$ surface in the $2 \mathrm{D}$ condition (2DCOND) and on a 3D surface in the 3D condition (3DCOND). The stimuli sets contain the animated talking head with 20 different gaze angles. The angles are equally spaced between -25 degrees and +13 degrees in the $3 \mathrm{D}$ models internal gaze angle (horizontal eyeball rotation in relation to skull) with 2 degree increments, where 0 degree rotation is when the eyes are looking straight forward. The angles between +13 degrees and +25 degrees were left out because of a programming error, but we found no indications that this asymmetry has any negative effects on the experimental results.

Five subjects were simultaneous employed in a within-subject design, where each subject judged each stimulus in the experiment. All five subjects had normal or corrected to normal eye sight.

\subsection{Method}

Before the experiment, the subjects were presented with an answer sheet, and the task of the experiment was explained: to point out, for each stimulus, which subject the gaze of the animated head is pointing at. The advantage of using subjects as gaze target is that this method provides perceptually, and communicatively, relevant gaze targets instead of using, for example, a spatial grid as in [19]. For each set of 20 stimuli, each of the seated subjects got an empty answer sheet with 20 answer lines indicating the position of all subjects. The subject enters a mark on one of the subjects indicating her decision. If the subject believed the head was looking beyond the rightmost or the leftmost subject, the subject entered the mark at the end of either of the two arrows to the right or left of the boxes that represent the subjects. 

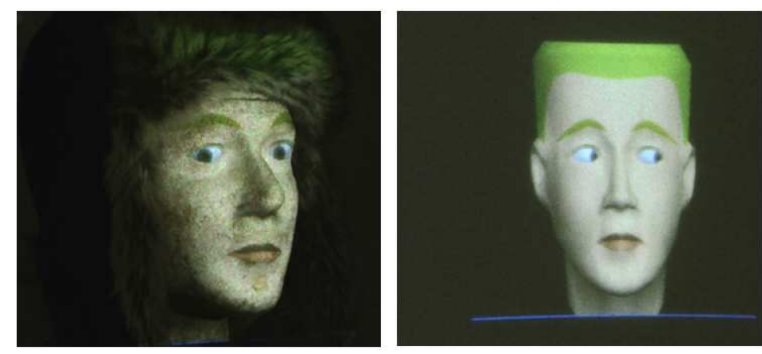

Fig. 5. Snapshots, taken over the shoulder of a subject, of the projection surfaces in 3DCOND (left) and 2DCOND (right).

The five subjects were then randomly seated at the five positions and the first set of 20 stimuli was projected in 3DCond, as seen on the left of figure 5 . Subjects marked their answer sheets after each stimulus. When all stimuli were presented, the subjects were shifted to new positions and the process repeated, in order to capture any bias for subject/position combinations. The process was repeated five times, so that each sat in each position once, resulting in five sets of responses from each subject.

\subsection{Analysis and Results}

Figure 6 plots the raw data for all the responses over gaze angles. The size of the bubbles indicates the number of responses with the corresponding value for that angle; the bigger the bubble, the more subjects perceived gaze in that particular direction. It is again clear that in 3DCond, the perception of gaze is more precise (i.e. fewer bubbles per row) compared to 2DCond.

Figure 7 shows bubble plots similar to those in figure 6 , with responses for each stimulus. The figure differs in that the data plotted is filtered so that only responses are plotted where perceived gaze matched the responding subject, that is when subjects responded that the gaze was directed directly at themselves what is commonly called eye-contact or mutual gaze. These plots show the location of and the number of the subjects that perceived eye-contact over different gaze angles. In 2DCond, the Mona Lisa gaze effect is very visible: for all the near-frontal angles, each of the five subjects, independently from where they are seated, perceived eye-contact. The figure also shows that the effect is completely eliminated in 3DCond, in which generally only one subject at a time perceived eye-contact with the head.

\subsection{Estimating the gaze function}

In addition to investigating the gaze perception accuracy of projections on different types of surfaces, the experimental setup allows us to measure a psychometric function for gaze which maps eyeball rotation in a virtual talking head to physical, real-world angles an essential function to establish eye-contact between 


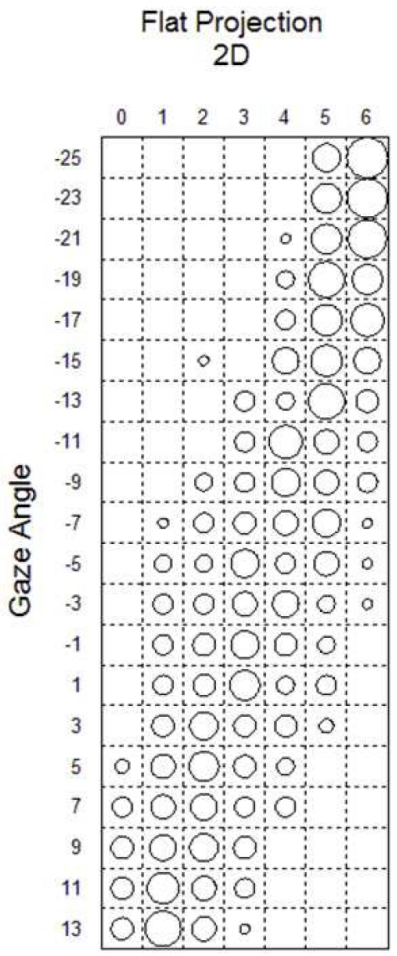

Gaze Decision
Head Model Projection

3D

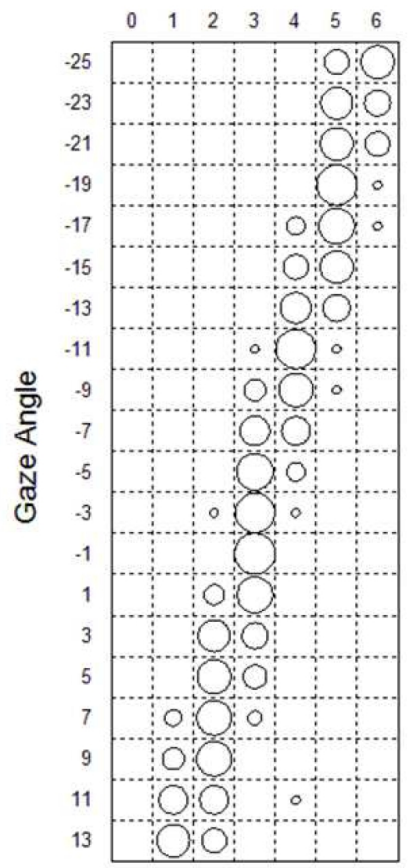

Gaze Decision

Fig. 6. Responses for all subject positions ( $X$ axis) over all internal angles ( $Y$ axis) for each of the conditions: 2DCOND to the left and 3DCOND to the right. Bubble size indicates number of responses. The $X$ axis contains the responses for each of the five subject positions (from 1 to 5), where 0 indicates gaze perceived beyond the leftmost subject, and 6 indicates gaze perceived beyond the rightmost subject.

the real and virtual world. We estimated this function by applying a first order polynomial fit to the data to get a linear mapping from the real positions of the gaze targets perceived by the subjects, to the actual internal eyeball angles in the projected animated talking head, for each condition.

In 2DCOND, the estimated function that resulted from the linear fit to the data is:

$$
\text { Angle }=-5.2 \times \text { Gaze Target }
$$

$$
\mathrm{RMSE}=17.66
$$

$\mathrm{R}$ square $=.668$ 


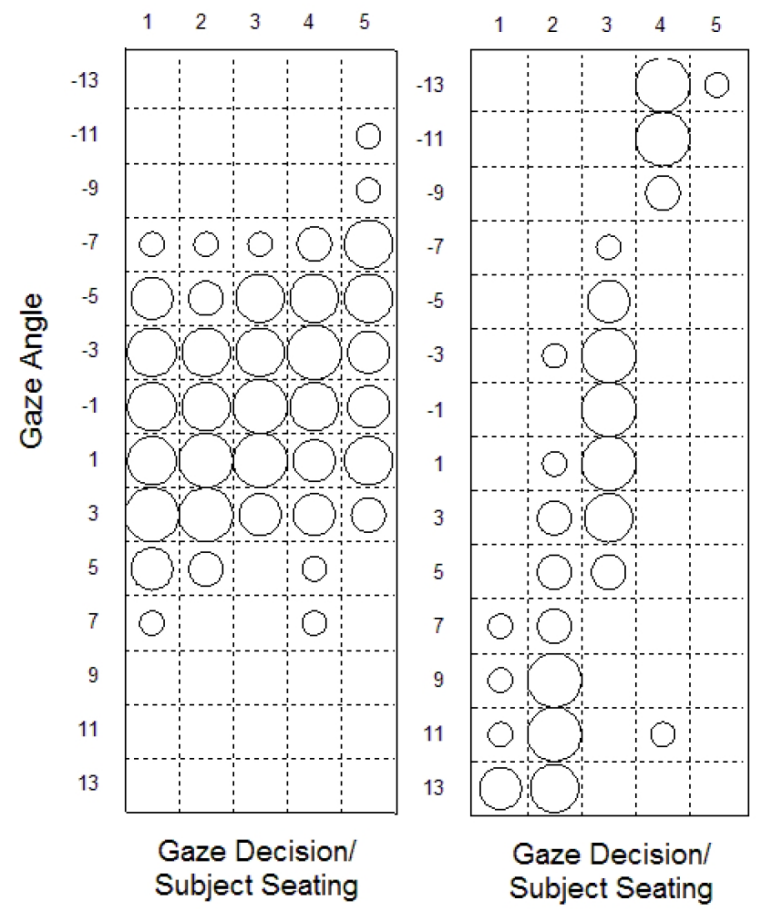

Fig. 7. Bubble plot showing only responses where subjects perceived eye-contact: subject position ( $X$ axis) over all internal angles ( $Y$ axis) for each of the conditions: 2DCond to the left and 3DCond to the right. Bubble size indicates number of responses.

And for the 3DCOND:

$$
\begin{gathered}
\text { Angle }=-4.1 \times \text { Gaze Target } \\
\text { RMSE }=6.65 \\
\text { R square }=.892
\end{gathered}
$$

where $\mathrm{R}$ square represents the ability of the linear fit to describe the data.

Although the resulting gaze functions from the two conditions are similar, the goodness of fit is markedly better in 3DCOND than in 2DCOND.

The results provide a good estimation of a gaze psychometric function. If the physical target gaze point is known, the internal angle of eye rotation can be calculated. By reusing the experimental design, the function can be estimated for any facial design or display surface.

\section{Spatial faithfulness of gaze and situated interaction}

Armed with this distinction between perception of gaze in 2D and 3D displays, we now turn to how communicative gaze requirements are met by the two system types. Situated interaction requires a shared perception of spatial properties 
where interlocutors and objects are placed, in which direction a speaker or listener turns, and at what the interlocutors are looking. Accurate gaze perception is crucial, but plays different roles in different kinds of communication, for example between co-located interlocutors, between humans in avatar or video mediated human-human communication, and between humans and ECAs or robots in spoken dialogue systems.

We propose that it is useful to talk about three levels of gaze faithfulness, as follows. We define the observer as the entity perceiving gaze and a target point as an absolute position in the observers space.

- Mutual Gaze. When the observer is the gaze target, the observer correctly perceives this. When the observer is not the gaze target, the observer correctly perceives this. In other words, the observer can correctly answer the question: Does she look me in the eye?

- Relative Gaze. There is a direct and linear mapping between the intended angle of the gaze relative to the observer and the observers perception of that angle. In other words, the observer can correctly answer the question: How much to the left of/to the right of/above/below me is she looking?

- Absolute Gaze. A one-to-one mapping is correctly preserved between the intended target point of gaze and the observers perception of that target point. In other words, the observer can accurately answer the question: At what exactly is she looking?

Whether a system can produce faithful gaze or not depends largely on four parameters. Two of these represent system capabilities: the type of display used, limited here to whether the system produces gaze on a 2D surface or on a 3D surface and whether the system knows where relevant objects (including the interlocutors head and eyes) are in physical space; e.g. through automatic object tracking or with the help of manual guidance). A special case of the second capability is the ability to know only where the head of the interlocutor is. The remaining two have to do with the requirements of the application: the first is what level of faithfulness is needed as discussed above and the second whether the system is to interact with one or many interlocutors at the same time.

We start by examining single user systems with a traditional 2D display without object tracking, These systems are faithful in terms of mutual gaze no matter where in the room the observer is the system can look straight ahead to achieve mutual gaze and anywhere else to avoid it; it is faithful in terms of relative gaze regardless of where in the room the observer is, the system can look to the left and be perceived as looking to the right of the observer, and so on; and it is unrealistic in terms of absolute gaze the system can only be perceived as looking at target objects other than the observer by pure luck.

Next, we note that single user systems with a traditional 2D display with object tracking are generally the same as those without object tracking. It is possible, however, that the object tracking can help absolute gaze faithfulness, but it requires a fairly complex transformation involving targeting the objects in terms of angles relative the observer. If the objects are targeted in absolute terms, the observer will not perceive gaze targets as intended. 


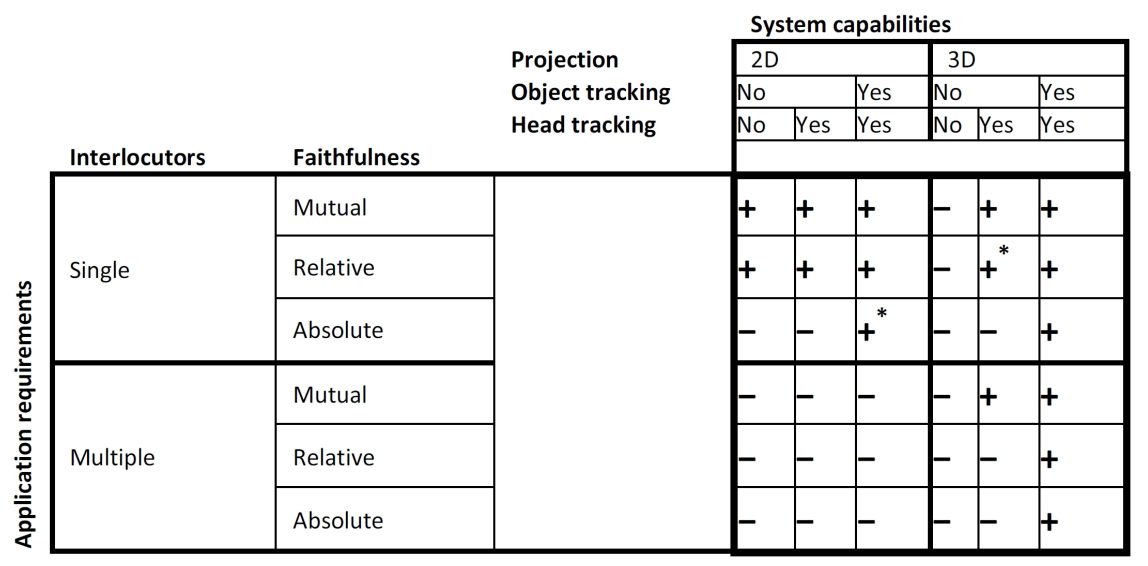

Fig. 8. Faithful (+) or unrealistic (-) gaze behaviour under different system capabilities and application requirements. $+{ }^{*}$ signifies that although faithfulness is most likely possible, it involves unsolved issues and additional transformations that are likely to cause complications.

Multi-user systems with a traditional 2D display and no object tracking perform poorly. They are unrealistic in terms of mutual gaze, as either all or none of the observers will perceive mutual gaze; they are unrealistic with respect to relative gaze, as all observers will perceive the gaze to be directed at the same angle relative themselves; and they are unrealistic in terms of absolute gaze as well.

Multi-user systems with a traditional 2D display and object tracking perform exactly as poorly as those without object tracking regardless of any attempt to use the object tracking to help absolute faithfulness by transforming target positions in relative terms, all observers will perceive the same angle in relation to themselves, and only one at best will perceive the intended position.

Turning to the $3 \mathrm{D}$ projection surface systems, both single and multi user systems with a $3 \mathrm{D}$ projection surface and no object tracking are unrealistic in terms of mutual gaze, relative gaze, and absolute gaze without knowing where to direct its gaze in real space, it is lost. By adding head tracking, the systems can produce faithful mutual gaze, and single user systems with head tracking can attempt faithful relative gaze by shifting gaze angle relative the observers head.

In contrast, both single and multi user systems with a 3D projection surface and object tracking, coupling the ability to know where objects and observers are with the ability to target any position, are faithful in terms of all of mutual gaze, relative gaze, and absolute gaze.

Figure 8 presents an overview of how meeting the three levels of faithfulness depends on system capabilities and application requirements. Examining the table in the figure, we first note that in applications where more than one 
participant is involved, using a 2D projection surface will result in a system that is unrealistic on all levels (lower left quadrant of the table), and secondly, that a system with a 3D projection surface and object tracking will provide faithful eye gaze regardless of application requirements (rightmost column). These are the perhaps unsurprising results of the Mona Liza gaze effect being in place in the first case, causing the gaze perception of all in a room to be the same, and of mimicking the conditions under which a situated human interacts in the second, with a physical presence in space and full perception of the environment and ones relation to it.

Thirdly, we note that if no automatic or manual object or head tracking is available, the 3D projection surface is unrealistic in all conditions, as it requires information on where in the room to direct its gaze, and that head only tracking improves the situation to some extent.

Fourthly, and more interestingly, we note that in single user cases where no object tracking or head tracking only is available, the $2 \mathrm{D}$ surface is the most faithful one (upper left quadrant). In these cases, we can tame and harness the Mona Lisa gaze effect and make it work for us. This suggests that gaze experiments such as those described in [20] and [21] could not have been performed with a 3D projection surface unless sophisticated head trackers would have been employed.

In summation, it is worthwhile to have a clear view of the requirements of the application or investigation before designing the system. In some cases (i.e. single user cases with no need for absolute gaze faithfulness), a simpler 2D display system without any tracking can give results similar to a more complex 3D projection surface system with head or object tracking facilities at considerably lower cost and effort. On the other hand, if we are to study situated interaction with objects and multiple participants, we need to guarantee successful delivery of gaze at all levels with a 3D projection surface that inhibits the Mona Lisa stare effect and reliable object tracking, manual or automatic, to direct the gaze.

\section{Applications and discussions}

As we have seen, the Mona Lisa gaze effect is highly undesirable in several communicative setups due to the manner in which it limits our ability to control gaze target perception. We have also seen that under certain circumstances, the effect a cognitive ability to perceive a depicted scene from the point of view of the camera or painter can be harnessed to allow us to build relatively simple applications, which would otherwise have required much more effort. A hugely successful example is the use of TV screens and movie theaters, where entire audiences perceive the same scene, independently from where they are seated. If this was not the case, the film and TV industries might well have been less successful. There are also situations where an ECA can benefit from establishing eye-contact with either all viewers simultaneously in a multiparty situation, as when delivering a message or taking the role of e.g. a weather presenter, and when it is required to establish eye contact with one person whose position in 
the room is unknown to the ECA, as is the case in most spoken dialogue system experiments to date involving an ECA.

Although the Mona Lisa gaze effect can be exploited in some cases, it is an obstacle to be overcome in the majority of interaction scenarios, as those where gaze is required to point exclusively to objects in the physical $3 \mathrm{D}$ space of the observer, or where multiple observers are involved in anything but the most basic interactions. In order to do controlled experiments investigating gaze in situated multiparty dialogues, the Mona Lisa effect must be overcome, and we can do this readily using the proposed technique. In other words, the technique opens possibilities for many applications which require absolute gaze perception, but would not have been possible with the use of a $2 \mathrm{D}$ display. In the following we present a short list of application families that we have recently begun to explore in the situated interaction domain, all of which require the levels of gaze perception afforded by $3 \mathrm{D}$ projection surfaces.

The first family of applications is situated and multiparty dialogues with ECAs or social conversational robots. These systems need to be able to switch their attention among the different dialogue partners, while keeping the partners informed about the status of the dialogue and who is being addressed, and exclusive eye-contact with single subjects is crucial for selecting an addressee. In such scenarios, a coherently shared and absolute perception of gaze targets is needed to achieve a smooth human-like dialogue flow a requirement that can not be met unless the Mona Lisa gaze effect is eliminated.

The second family involves any application where there is a need for a pointing device to point at objects in real space the space of the human participant. Gaze is a powerful pointing device that can point from virtual space to real space while being completely non-mechanic as opposed to for example fingers or arrows and it is non-intrusive and subtle.

A third family of applications is mediated interaction and tele-presence. A typical application in this family is virtual conferential systems. In a traditional system, the remote partner cannot meaningfully gaze into the environment of the other partners, since the remote partner is presented through a $2 \mathrm{D}$ display subject to the Mona Lisa gaze effect. Establishing a one-to-one interaction through mutual gaze cannot be done, as there is no ability to establish an exclusive eye contact. In addition to that, people look at the video presenting the other partners instead of looking into the camera, which is another obstacle for shared attention and mutual gaze and no one can estimate reliably at what the remote participant is looking. If a $3 \mathrm{D}$ head is used to represent the remote subject, who is represented through mediation as an avatar, these limitations to video conferential can, at lease partially, be resolved.

\section{Conclusions}

To sum up, we have proposed two ways of taming Mona Lisa: firstly by eliminating the effect and secondly by harnessing and exploiting it. 
En route to this conclusion, we have proposed an affordable way of eliminating the effect by projecting an animated talking head on a 3D projection surface a generic physical 3D model of a human head, and verified experimentally that it allows subjects to perceive gaze targets in the room clearly from various viewing angles, meaning that the Mona Lisa effect is eliminated. In the experiment, the $3 \mathrm{D}$ projection surface was contrasted with a $2 \mathrm{D}$ projection surface, clearly displaying the Mona Lisa gaze effect in the 2D case. In addition to eliminating the Mona Lisa gaze effect, the 3D setup allowed observers to perceive with very high agreement who was being looked at. The $2 \mathrm{D}$ setup showed no such agreement. We showed how the data serves to estimate a gaze psychometric function to map actual gaze target into eyeball rotation values in the animated talking head.

Based on the experimental data and the working model, we proposed three levels of gaze faithfulness relevant to applications using gaze: mutual gaze faithfulness, relative gaze faithfulness, and absolute gaze faithfulness. We further suggested that whether a system achieves gaze faithfulness or not depends on several system capabilities whether the system uses a $2 \mathrm{D}$ display or the proposed 3D projection surface, and whether the system has some means of knowing where objects and the interlocutors are, but also depending on the application requirements whether the system is required to speak to more than one person at a time and the level of gaze faithfulness it requires.

One of the implications of this is that the Mona Lisa gaze effect can be exploited and put to work for us in some types of applications. Although perhaps obvious, it falls out neatly from the working model. Another implication is that the only way to robustly achieve all three levels of gaze faithfulness is to have some means of tracking objects in the room and to use an appropriate 3D projection surface. However, without knowledge of objects positions, the 3D projection surface falls short.

We close by discussing the benefits of 3D projection surfaces in terms of human-robot interaction, where the technique can be used to create faces for robotic heads with a high degree of human-likeness, better design flexibility, more sustainable animation, low weight and noise levels and lower maintenance costs, and by discussing in some detail a few application types and research areas where the elimination of the Mona Lisa gaze effect through the use of $3 \mathrm{D}$ projection surfaces is particularly useful, such as when dealing with situated interaction or multiple interlocutors.

We consider this work to be a stepping stone for several future investigations and studies into the role and employment of gaze in human-robot, human-ECA, and human-human mediated interaction.

Acknowledgments. This work has been partly funded by the EU project IURO (Interactive Urban Robot) FP7-ICT-248314 . The authors would like to thank the five subjects for participating in the experiment. 


\section{References}

1. Beskow, J., Edlund, J., Granstrm, B., Gustafson, J., \& House, D. :Face-to-face interaction and the KTH Cooking Show. In Esposito, A., Campbell, N., Vogel, C., Hussain, A., \& Nijholt, A. (Eds.), Development of Multimodal Interfaces: Active Listening and Synchrony (pp. 157 - 168). Berlin / Heidelberg: Springer, (2010).

2. Ruttkay, Z. \& Pelachaud, C. (editors) :From Brows till Trust: Evaluating Embodied Conversational Agents, Kluwer, (2004).

3. Pelachaud, C. Modeling Multimodal Expression of Emotion in a Virtual Agent. Philosophical Transactions of Royal Society B Biological Science, B 2009 364, 35393548, (2009).

4. Granstrm, B., \& House, D. :Modeling and evaluating verbal and non-verbal communication in talking animated interface agents. In Dybkjaer, 1., Hemsen, H., \& Minker, W. (Eds.). Evaluation of Text and Speech Systems (pp. 65-98). Springer-Verlag Ltd, (2007).

5. Shinozawa K., Naya F., Yamato J., Kogure K. :Differences in effect of robot and screen agent recommendations on human decision-making. International Journal of Human Computer Studies, 62 (2), (pp. 267-279), (2005).

6. Todorovi, D. :Geometrical basis of perception of gaze direction. Vision Research, 45(21), (pp. 3549-3562), (2006).

7. Gockley, R., Simmons, J., Wang, D., Busquets, C., DiSalvo, Ke., Caffrey, S., Rosenthal, J., Mink, S., Thomas, W., Adams, T., Lauducci, M., Bugajska, D., Perzanowski, and A. Schultz. :Grace and George: Social Robots at AAAI. Proceedings of AAAI'04. Mobile Robot Competition Workshop, pp. 15-20, AAAI Press, (2004).

8. Sosnowski, S., Mayer, C., Kuehnlenz, K., Radig, B. :Mirror my emotions! Combining facial expression analysis and synthesis on a robot. Proceedings of the Thirty Sixth Annual Convention of the Society for the Study of Artificial Intelligence and Simulation of Behaviour (AISB10), (2010).

9. Raskar, R., Welch, G., Low, K-L., \& Bandyopadhyay, D. :Shader lamps: animating real objects with image-based illumination. In Proc. of the 12th Eurographics Workshop on Rendering Techniques (pp. 89-102), (2001).

10. Lincoln, P., Welch, G., Nashel, A., Ilie, A., State, A., \& Fuchs, H. :Animatronic shader lamps avatars. In Proc. of the 2009 8th IEEE International Symposium on Mixed and Augmented Reality (ISMAR '09). Washington, DC, US: IEEE Computer Society, (2009).

11. Beskow, J. :Talking heads Models and applications for multimodal speech synthesis. Doctoral dissertation, KTH, (2003).

12. Parke, F. I. Parameterized Models for Facial Animation. Computer Graphics and Applications, IEEE, 2(9), (pp. 61-68), (1982).

13. Kendon, A. :Some functions of gaze direction in social interaction. Acta Psychologica, 26, (pp. 22-63), (1967).

14. Argyle, M., \& Cook, M. :Gaze and mutual gaze. ISBN: 978-0521208659, Cambridge University Press, (1976).

15. Kleinke, C. L. :Gaze and eye contact: a research review. Psychological Bulletin, 100, 78-100, (1986).

16. Takeuchi, A., \& Nagao, K. Communicative facial displays as a new conversational modality. In Proc. of the INTERACT '93 and CHI '93 conference on Human factors in computing systems, (1993). 
17. Bilvi, M., \& Pelachaud, C. :Communicative and statistical eye gaze predictions. In Proc. of International conference on Autonomous Agents and Multi-Agent Systems. Melbourne, Australia, (2003).

18. Gregory, R. :Eye and Brain: The Psychology of Seeing. Princeton: Princeton University Press, (1997).

19. Delaunay, F., de Greeff, J., \& Belpaeme, T. :A study of a retro-projected robotic face and its effectiveness for gaze reading by humans. In Proc. of the 5th ACM/IEEE international conference on Human-robot interaction, ACM (pp. 39-44), (2010).

20. Edlund, J., \& Nordstrand, M. :Turn-taking gestures and hour-glasses in a multimodal dialogue system. In Proc. of ISCA Workshop on Multi-Modal Dialogue in Mobile Environments. Kloster Irsee, Germany, (2002).

21. Edlund, J., \& Beskow, J. :MushyPeek - a framework for online investigation of audiovisual dialogue phenomena. Language and Speech, 52(2-3), (pp.351-367), (2009). 